\title{
Black Hole Binary Mergers
}

\author{
Junichiro Makino \\ Department of Astronomy, School of Science, The University of Tokyo, \\ 7-3-1 Hongo, Bunkyo-ku, Tokyo 113-0033, Japan
}

\begin{abstract}
.
I overview the current understanding of the evolution of massive black hole (MBH) binaries in the center of the host stellar system. One of the main questions is whether the stellar dynamical effect can make the MBH binary hard enough that they can merge through gravitational wave radiation. So far, theories and numerical simulations suggested otherwise, since the hardening time scale becomes very long once "loss cone" is depleted. I'll present the result of recent simulations on this hardening time scale, and discuss its implication on the formation history of massive black holes.
\end{abstract}

\section{Introduction}

There are now plenty of evidences that many, if not most, of giant ellipticals contain massive central black holes (Magorrian et al. 1998). Also, it has been suggested that the black hole mass $M_{\mathrm{BH}}$ shows tight correlation with the spheroidal mass and the central velocity dispersion (Gebhardt et al. 2000; Ferrarese \& Merritt 2000). The most straightforward explanation of such correlation is that massive galaxies are formed by merging of less massive galaxies, and that the central black hole also grows through merging (Kauffmann \& Haehnelt 2000). This merging scenario naturally explains the observed correlations.

This merging scenario has additional important characteristic that it nicely explains the observed structure of the central region of massive elliptical galaxies. High-resolution observations by HST revealed that the "cores" of the giant elliptical galaxies are not really cores with flat volume density, but very shallow cusps expressed as $\rho \propto r^{-\alpha}$, where $\rho$ is the volume luminosity density and $r$ is the distance from the center, with power index $\alpha=0.5 \sim 1$ Gebhardt et al. (1996), Byun et al. (1996). Makino \& Ebisuzaki (1996) performed the simulation of repeated mergers of galaxies with central black holes. They found that the structure of the merger converges to one profile. The merger product has a central cusp around the black hole with slope approximately -0.5 , and the total mass of the stars in the cusp region is around the mass of the black hole binary. Nakano \& Makino (1999b), Nakano \& Makino (1999a) showed, by combination of simple $N$-body simulation and an analytic argument, that this shallow cusp is explained by the fact that distribution function of stars has a lower cutoff energy $E_{0}$. Their analytic argument naturally explains the observed correlation between the cusp radius and the black hole mass.

One remaining problem is what will happen to the binary black hole. Begelman et al, (1980) [hereafter BBR] predicted that the hardening of the BH binary 
would be halted once it ejected all the stars in its "loss cone". Early numerical simulations Makino et al. (1993), Mikkola \& Valtonen (1992) could not cover the range of the number of particles (relaxation time) wide enough to see the change in the time scale. Recent studies Makino (1997), Quinlan \& Hernquist (1997), Chatterjee et al. (2003), Milosavljevic \& Merritt (2001) reported the results, which are not only mutually inconsistent but also inconsistent with the theoretical prediction. BBR predicted that after the stars in the loss cone is depleted, the hardening time scale would be the relaxation time scale of the parent galaxy, since in the relaxation time scale stars would diffuse into the loss cone. The results of numerical simulation ranges from no dependence on the relaxation time scale (Milosavljevic \& Merritt 2001), to some dependence with upper limit in the time scale (Quinlan \& Hernquist 1997; Chatterjee et al. 2003), and finally to weaker-than-linear dependence (Makino 1997).

Thus, it has been an open question whether or not the loss-cone depletion occurs. Some even argued that numerical simulations would not help in determining the fate of a $\mathrm{BH}$ binary. Here, we present the result of our resent $N$-body simulations with up to $1 \mathrm{M}$ particles, where we saw a clear sign of the loss-cone depletion.

\section{Numerical Method and Initial Models}

The details of the model is given elsewhere (Makino \& Funato 2004). We performed $N$-body simulations using direct summation method on GRAPE-6 (Makino et al 2002). Gravitational interaction between field particles is softened, while that involves BH particles is not.

The initial galaxy model is a King model with non-dimensional central potential of $W_{0}=7$. We use the Heggie unit, where the mass $M$ and the virial radius $R_{v}$ of the initial galaxy model and gravitational constant $G$ are all unity. The mass of black hole particles is $M_{B H}=0.01$. They are initially placed at $( \pm 0.5,0,0)$ with velocity $(0, \pm 0.1,0)$.

The largest calculation ( 1 million particles and up to $t=400$ ) took about one month, on a single-host, 4-processor-board GRAPE-6 system with the peak speed of 4 Tflops.

\section{Results}

Figure 1 shows the time evolution of the specific binding energy (per reduced mass) $E_{b}$ of the black hole binary. It is clear that the hardening time scale becomes longer as we increase $N$ (and thereby the relaxation time).

To quantitatively evaluate the dependence of the hardening rate on the number of particles, we calculated the hardening rate $\beta$ (see figure 1). When $E_{b}=-1$, the hardening rate is almost independent of $N$. However, as the binary becomes harder, $\beta$ decreases, and the decrease is larger for larger $N$. Thus, the hardening rate $\beta$ for large values of $\left|E_{b}\right|$ shows strong dependence on the number of particles $N$. For $E_{b}=-7$, the hardening rate $\beta$ is almost proportional to $1 / N$. In other words, we obtained the result which is consistent with the theory. The hardening time scale is proportional to the relaxation time scale of the parent galaxy. 

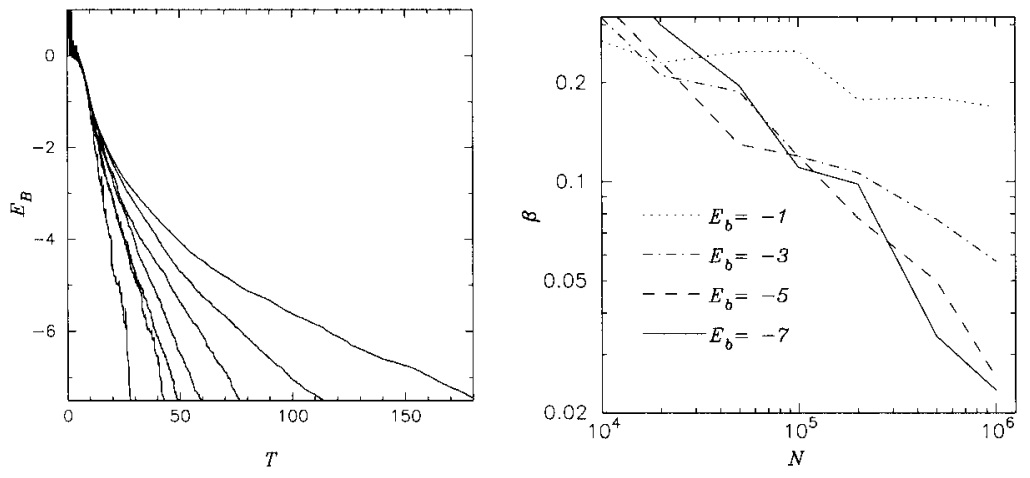

Figure 1. (Left) the evolution of the specific binding energy $E_{b}$ of the binary black hole. Curves are result with $N=10 \mathrm{~K}, 20 \mathrm{~K}, 50 \mathrm{~K}, 100 \mathrm{~K}, 200 \mathrm{~K}, 500 \mathrm{~K}$ and $1 \mathrm{M}$ (left to right). For all calculations, the softening length is $\epsilon=0.01$. (Right) the hardening rate $\beta=\left|\Delta E_{b} / \Delta t\right|$ plotted as a function of the number of particles $N$. Dotted, dash-dotted, dashed and solid curves denote the values measured from the time at which $\left|E_{b}\right|$ reached $1,3,5$ and 7 , respectively. The end time is the time at which $\left|E_{b}\right|$ reached initial value +0.5 .

\section{Discussions}

Our present result is consistent with the theory of loss-cone depletion and its refilling in thermal time scale, while previous numerical results are not. What caused this difference?

Makino (1997) obtained the dependence much weaker than linear from simulations with $N=2,048$ to 266,144 . If we compare his figure 1 and our figure 1 , the reason is obvious. The value of the binding energy at which the dependence is measured was rather small. In other words, Makino (1997) did not cover long enough time.

Quinlan \& Hernquist (1997) performed simulations very similar to the ones presented here. They found that the hardening rate was practically the same for $N=10^{5}$ and $N=2 \times 10^{5}$. We do not really understand why they obtained this result. They used the SCF method by Hernquist \& Ostriker (1992) and they varied the masses of particles depending on their initial angular momenta. We suspect this combination might have complicated the dependence of the hardening time scale on the number of particles. Chatterjee et al (2003) might have similar problem.

Milosavljevic \& Merritt (2001) performed three runs with 8K, 16K and $32 \mathrm{~K}$ stars, and found that the hardening rate was independent of the number of particles. As suggested in their paper, this result is simply because the loss cone was not depleted in their simulation, primarily because $N$ was too small. So their result is not inconsistent with ours.

As first suggested by BBR and confirmed by a number of followup works, if the hardening time scale of the black hole binary is proportional to the relaxation time scale of the parent galaxy, the evolution time scale of a typical binary black hole in an elliptical galaxy exceeds the Hubble time by many orders of 
magnitude. In other words, the binary is unlikely to merge through encounters with field stars and gravitational wave radiation.

Our result strongly suggests that the hardening time scale is indeed determined by the relaxation time scale, for large enough $N$ and after the binary becomes sufficiently hard. Thus, our result imply that gravitational interaction with field stars is insufficient to let the binary merge.

If the binary black hole has long lifetime, it is quite natural to assume that some of the host galaxies which contain binary black holes would further merge with another galaxy with a central massive black hole or a binary. Simple estimate assuming the thermal distribution of the eccentricity Makino \& Ebisuzaki (1994) suggests that, during repeated three body interactions, the eccentricity of the binary can reach a very high value, resulting in quick merging through gravitational wave radiation. Thus, strong triple interaction of three super-massive black holes may be common.

\section{References}

Begelman, M. C., Blandford, R. D., \& Rees, M. J. 1980, Nature, 287, 307

Byun, Y.-I., et al. 1996, AJ, 111, 1889

Chatterjee, P., Hernquist, L., \& Loeb, A. 2003, ApJ, 592, 32

Ferrarese, L., \& Merritt, D. 2000, ApJ, 539, L9

Gebhardt, K., et al. 2000, ApJ, 539, L13

Gebhardt, K., et al. 1996, AJ, 112, 105

Kauffmann, G., \& Haehnelt, M. 2000, MNRAS, 311, 576

Hernquist, L., \& Ostriker, J. P. 1992, ApJ, 386, 375

Magorrian, J., et al. 1998, AJ, 115, 2285

Makino, J. 1997, ApJ, 478, 58

Makino, J., \& Ebisuzaki, T. 1994, ApJ, 436, 607

—. 1996, ApJ, 465, 527

Makino, J., et al. 2002, ASP Conf. Proc. 263, 389

Makino, J., \& Funato, Y. 2003, ApJ, 602, 93

Makino, J., et al. 1993, PASJ, 45, 303

Mikkola, S., \& Valtonen, M. J. 1992, MNRAS, 259, 115

Milosavljević, M., \& Merritt, D. 2001, ApJ, 563, 34

Nakano, T., \& Makino, J. 1999a, ApJ, 525, L77

-. 1999b, ApJ, 510, 155

Quinlan, G. D., \& Hernquist, L. 1997, New Astronomy, 2, 533 